\title{
Two-Junction Tuning Circuits for Submillimeter SIS Mixers
}

\author{
Jonas Zmuidzinas, Henry G. LeDuc, Jeffrey A. Stern, and Scott R. Cypher
}

\begin{abstract}
The capacitance of superconducting tunnel junctions can seriously degrade the performance of quasiparticle (SIS) mixers operating in the submillimeter band, so it is essential to provide a circuit for tuning out this capacitance at the operating frequency. In this article, we present two new tuning circuits for SIS mixers which use a pair of SIS junctions connected by an inductance. Compared to previously proposed tuning circuits, ours have a broader bandwidth, are easier to scale to higher frequencies, and may be easier to fabricate. We have constructed quasi-optical mixers which employ these tuning circuits, using $\mathrm{Nb} / \mathrm{Al}$-Oxide/Nb SIS junctions defined by optical lithography. The performance of these devices is excellent, giving receiver noise temperatures of $113 \mathrm{~K}$ (DSB) at $490 \mathrm{GHz}$ and $230 \mathrm{~K}$ DSB at $612 \mathrm{GHz}$. In addition to demonstrating the effectiveness of our tuning circuit, these results show that quasi-optical mixers can be competitive with or superior to waveguide mixers at submillimeter wavelengths. The mixers continue to perform well at frequencies up to $672 \mathrm{GHz}$, which is about $95 \%$ of the $\mathrm{Nb}$ gap frequency.
\end{abstract}

\section{INTRODUCTION}

$\mathbf{P}$ REVIOUSLY reported SIS mixers have shown very lownoise performance at frequencies up to about $500 \mathrm{GHz}$. Several recent reviews [1], [2], have summarized the experimental status of SIS receivers. Typically, excellent results have been obtained by using a waveguide mount whose backshorts are carefully tuned to optimize the match to the highly capacitive SIS junction. At higher frequencies, the admittance of the junction capacitance grows but meanwhile the backshort and waveguide will inevitably suffer increasing losses which reduce the effectiveness of the tuning. Also, the resulting high- $Q$ resonance implies a small instantaneous bandwidth, so the backshorts must be retuned if the operating frequency is changed. To alleviate these problems, a lithographic tuning circuit is often incorporated with the SIS junction in order to provide compensation for the junction capacitance at the operating frequency, as first described in [3], [4] and further discussed in the reviews [1], [2]. The tuning circuit relaxes the severe constraints placed on the backshort and waveguide losses, and allows the construction of "fixed-tuned" or "tunerless" mixers. The instantaneous bandwidth of such mixers is

Manuscript received May 26, 1993; revised August 23, 1993.This work was supported in part by NASA under Grants NAGW-107 and NAG2-744, and NASA/JPL, and an NSF Presidential Young Investigator grant, amd in part by the Strategic Defense Initiative Organization (Innovative Science and Technology Office) and the National Aeronautics and Space Administration (Office of Advanced Concepts and Technology).

J. Zmuidzinas is with the George W. DownsLaboratory of Physics, California Institute of Technology, Pasadena, CA 91125.

H. G. LeDuc, J. A. Stern, and S. R. Cypher are with Jet Propulsion Laboratory, 302-231, Pasadena, CA.

IEEE Log Number 9216062. often broad enough to allow the backshort to be kept at one fixed setting, which greatly simplifies the construction and operation of the receiver and improves reliability.

Quasi-optical techniques provide a convenient alternative to waveguide mixers. A quasi-optical SIS mixer (e.g. [5]-[10]) generally consists of an SIS junction substrate mounted onto a lens, typically a hyperhemisphere or ellipsoid. The lens serves to focus the radiation onto a microantenna which has been lithographically defined and integrated with the SIS junction. In comparison to waveguide mixers, quasi-optical mixers are far easier and less expensive to fabricate and would be much more amenable to integration in a focal-plane array. However, because quasi-optical mixers lack an adjustable element equivalent to a waveguide backshort, integrated tuning circuits are essential in order to achieve competitive performance. Although quasi-optical receivers with tuning circuits have previously been constructed for the millimeter band ([7], [8]), tuned mixers for the submillimeter band are a recent development. Büttgenbach et al. [10] have demonstrated a quasi-optical mixer which incorporated SIS junctions, a tuning circuit, and a planar spiral antenna. Using this mixer, a receiver noise temperature of $220 \mathrm{~K}$ (DSB) was measured at $426 \mathrm{GHz}$, which was competitive with results obtained using waveguide receivers.

It is clear from the discussion above that tuning circuits will play a critical role in extending the performance of both waveguide and quasi-optical SIS mixers to frequencies above $500 \mathrm{GHz}$. Although many different tuning circuit designs have been proposed to date, we will show that it may be difficult to scale these designs to higher frequencies without compromising bandwidth or performance. This difficulty motivated us to try a new approach to the problem, namely to use two SIS junctions coupled to each other through a small microstrip inductance. We give an analysis of two such configurations, and argue that this approach offers many advantages, including broad bandwidth, ease of fabrication, precise control of the tuning inductance, and straightforward scaling to higher frequencies. We have fabricated and tested quasi-optical slot antenna mixers using these two-junction tuning circuits, and have obtained excellent results, which are competitive with or superior to the best waveguide results reported to date.

\section{COnventional Tuning Circuits For SIS MLXers}

Almost all of the SIS tuning circuits proposed to date fall into two broad categories, depending on whether a tuning inductance is placed in series or in parallel with the junction. 


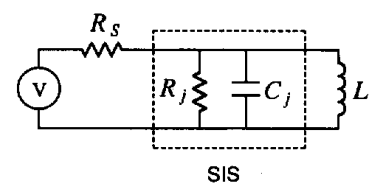

(a)

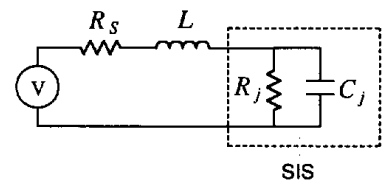

(b)

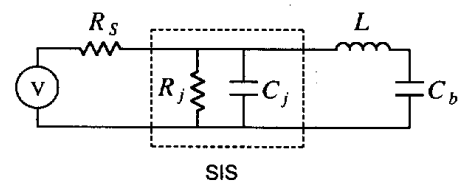

(c)

Fig. 1. Circuit diagrams of SIS junctions with: (a) a shunt tuning inductance; (b) a series tuning inductance; and (c) a shunt inductance $L$ in series with a blocking capacitance $C_{b}$ to allow d.c. biasing.

For instance, a $3 / 8 \lambda$ open-circuit microstrip stub tuner ([4]) can be thought of as a $\lambda / 8$ microstrip inductance placed in parallel with the junction by using a $\lambda / 4$ stub as an RF short circuit. In the sections below, we discuss these two categories of tuning elements before describing the two-junction circuits in section III.

\section{A. Junction Equivalent Circuit}

The geometric capacitance of an SIS junction can be represented by a capacitance $C_{j}$ which is connected in parallel with the quasiparticle tunneling impedance of the junction, $Z_{j}$. In general, $Z_{j}$ must be calculated from complex expressions for the tunneling current (e.g. [11], [12]) which give both real and imaginary components, but in many cases it is sufficient to approximate $Z_{j}$ by the junction normal-state resistance $R_{N}$.

\section{B. Parallel Shunt Inductance Tuning}

As shown in Fig. 1(a), the ideal tuning circuit for an SIS junction would simply consist of a lumped-element inductor $L$ connected in parallel with the junction, so that the admittances of the junction capacitance $j \omega C_{j}$ and tuning inductance $1 / j \omega L$ would cancel at the resonance frequency $\omega_{0}=1 / \sqrt{L C_{j}}$, leaving only the quasiparticle tunneling admittance. The tuning inductance is most often realized as an electrically short section of superconducting microstrip line. The bandwidth of this circuit is proportional to $1 / R_{N} C_{j}$. Note that the $R_{N} C_{j}$ product of the junction is a parameter that is independent of the junction area but which does depend on the current density roughly as $1 / J_{c}$. Thus, high current density junctions are needed to obtain broad bandwidths.

\section{Series Inductance Tuning and "End-loaded" Stubs}

Another possible tuning circuit is shown in Fig. 1(b), in which a series inductance is used. This is essentially the circuit proposed in [10], and is commonly referred to as an "endloaded" stub. Although this circuit is an excellent choice for frequencies below $500 \mathrm{GHz}$, it is less well suited to higher frequencies. The difficulty presented by this circuit is that it also acts as an impedance transformer, producing a real impedance on the order of $R_{N} /\left(\omega_{0} R_{N} C_{j}\right)^{2}$. At $500 \mathrm{GHz}$, we calculate $\omega_{0} R_{N} C_{j} \approx 5$ for $\mathrm{Nb} / \mathrm{Al}$-Oxide/ $\mathrm{Nb}$ junctions with $J_{c} \approx 10 \mathrm{kA} \mathrm{cm}{ }^{-2}$, so the transformed impedance is very low, about $1 \Omega$ for a junction area of $1 \mu \mathrm{m}^{2}$. A multisection microstrip quarter-wave transformer is then needed to bring the impedance back up to match the antenna. The transformer will reduce the bandwidth and make the circuit more susceptible to losses, especially at higher frequencies for which a larger transformation factor will be needed. In addition, this circuit rapidly becomes difficult to design at higher frequencies. Because of the very low impedance needed, the last section of the transformer becomes quite wide, and in fact the width can become comparable to the length. In addition, a large discontinuity in width is developed between this section and the series tuning inductor. This means that the traditional analysis based on transmission-line formulas is inadequate to accurately characterize the circuit, as are approximations which treat the discontinuities in terms of parasitic lumped elements as is common in microwave CAD programs. A more sophisticated method is needed which can calculate the 2dimensional distribution of current. Thus, the shunt inductance technique would appear to be the better approach, but this method also poses its own problems as we shall see.

\section{The Blocking Capacitance}

In practice, the shunt inductance tuning circuit of Fig. 1(a) must be modified by adding a blocking capacitance $C_{b}$ in series with the tuning inductance $L$ to allow a d.c. bias to be applied to the junction, as shown in Fig. 1c. In this section, we will outline some of the difficulties caused by this simple element. Some of these considerations have been discussed previously in [13], although not in detail.

The blocking capacitance (or more correctly, its function) can be implemented in several ways, for instance by a quarterwavelength section of open-circuited microstrip line or a radial stub. However, in order to avoid reducing the bandwidth, the RF impedance of this d.c blocking element must be kept small, $Z_{b}(\omega) \ll 1 / \omega C_{j}$. This constraint proves to be difficult to satisfy in practice for frequencies above $500 \mathrm{GHz}$. The essence of this difficulty is that an SIS junction is itself an excellent capacitor, as the junction is just two superconductors separated by a tunneling barrier around $10 \AA$ thick. The capacitance $C_{j}$ of the junction is given by the parallel-plate expression,

$$
C_{j}=88.5 \epsilon_{j} A_{j} / t_{j} \mathrm{fF},
$$

where $\epsilon_{j}$ is the relative dielectric constant of the $\mathrm{Al}_{2} \mathrm{O}_{3}$ tunneling barrier $\left(\epsilon_{j} \approx 10\right), A_{j}$ is the junction area in units of $\mu \mathrm{m}^{2}$, and $t_{j}$ is the barrier thickness measured in $\AA$. Experimentally, we find $C_{j} / A_{j} \approx 50-80 f \mathrm{~F} \mu \mathrm{m}^{-2}$ for $\mathrm{Nb} / \mathrm{Al}-$ Oxide $/ \mathrm{Nb}$ junctions with $J_{c} \approx 10 \mathrm{kA} \mathrm{cm}^{-2}$. The blocking capacitance $C_{b}$ must be made large in comparison to the junction capacitance $C_{j}$ in order to achieve the maximum 
bandwidth for the tuning circuit. For instance, if $C_{b}=2 C_{j}$, the resulting bandwidth is only $68 \%$ of the ideal case (i.e. $C_{b}=\infty$ ), while one obtains $91 \%$ of the full bandwidth for $C_{b}=10 C_{j}$.

The blocking capacitance can be fabricated by depositing a thin insulating film of dielectric constant $\epsilon_{b}$ and thickness $t_{b}$ between two superconducting layers. The capacitance is again given by the same parallel-plate formula, which can be used to express the required area $A_{b}$ :

$$
\frac{A_{b}}{A_{j}}=\frac{C_{b}}{C_{j}} \times \frac{t_{b}}{t_{j}} \times \frac{\epsilon_{j}}{\epsilon_{b}} .
$$

Equivalently, the ratio of linear dimensions, $l_{b} / l_{j}$, is given by the square root of this factor. If one assumes that the dielectric in the blocking capacitor is made with the $2000 \AA$ thick SiO or $\mathrm{SiO}_{2}$ films used in standard fabrication processes, one can easily see that the ratio of dimensions must be $l_{b} / l_{j} \approx 50$. Since SIS junctions typically have $l_{j} \approx 1 \mu \mathrm{m}$, the dimensions of the blocking capacitor cannot be kept small in comparison to the propagation wavelength and the capacitor must be treated as a distributed structure. In fact, the ratio of $l_{b}$ to the propagation wavelength $\lambda_{b}$ on the capacitor (which is essentially a superconducting microstrip line) can be expressed as

$$
\frac{l_{b}}{\lambda_{b}}=\sqrt{\frac{C_{b}}{C_{j}} \frac{\left(t_{b}+2 \lambda\right)}{t_{j}} \epsilon_{j}} \times \frac{l_{j}}{\lambda_{0}},
$$

where $\lambda$ is the London penetration depth and $\lambda_{0}$ is the free-space wavelength. Assuming $\epsilon_{j}=10, t_{j}=10 \AA$, $C_{b} / C_{j}=10, t_{b}=2000 \AA$ for the dielectric thickness, and $\lambda=700 \AA$ for the penetration depth of $\mathrm{Nb}$ films, we obtain $l_{b} / \lambda_{b}=184 l_{j} / \lambda_{0}$. Of course, the size of the capacitor can be reduced by using a very thin dielectric layer if one is willing to add another step in the fabrication process. However, in the limit $t_{b} \ll 2 \lambda$, we still have $l_{b} / \lambda_{b}=118 l_{j} / \lambda_{0}$. Even with a submicron junction having $l_{j}=0.5 \mu \mathrm{m}$, one finds that $l_{b} / \lambda_{b} \approx 0.1-0.2$ at $600 \mathrm{GHz}$, which clearly demonstrates the distributed nature of the blocking capacitor. For typical $\mathrm{NbN}$ films with $\lambda \approx 2500 \AA$, the effect is even worse: $l_{b} / \lambda_{b} \approx 0.2-0.3$. It is also very interesting to note that the junction itself may not be treated as a lumped element at very high frequencies. Indeed, one easily finds that

$$
\frac{l_{j}}{\lambda_{j}}=\sqrt{\left(1+2 \lambda / t_{j}\right) \epsilon_{j}} \times \frac{l_{j}}{\lambda_{0}}
$$

where $\lambda_{j}$ is the propagation wavelength inside the junction, ignoring the tunneling currents. As an example, in this paper we give measurements at $650 \mathrm{GHz}$ for mixers with junction sizes of $l_{j}=1.5 \mu \mathrm{m}$, for which $l_{j} / \lambda_{j} \approx 0.1$ ! The distributed nature of the junction may affect the detailed behavior of our mixer, and will need to be considered in future high frequency SIS mixer designs.

Since the blocking "capacitor" is not a true lumped element, one must consider the distribution of current across its surface. The current is injected in a small region (dictated by the width of the tuning inductor) and will spread out across the face of the capacitor. The associated "spreading inductance" reduces the effectiveness of the blocking capacitor which is reflected in a narrower tuning circuit bandwidth. The effect of this spreading inductance can be partially compensated for by increasing the size of the capacitor. For instance, a lossless radial stub can be designed to give a perfect short circuit at some frequency, and in fact radial stubs are often used in cases that a good broadband RF short-circuit is needed [14]. However, we have performed calculations of tuning circuits which include radial stubs as the RF short circuit/d.c. block element, and find that the frequency-dependent impedance of the radial stub does indeed reduce the bandwidth. The magnitude of this effect increases with frequency, junction area, and penetration depth. At $500 \mathrm{GHz}$ for $\mathrm{Nb}$ circuits, we typically find a $30 \%$ bandwidth reduction for junction areas around $1 \mu \mathrm{m}^{2}$.

\section{E. The Lithographic Registration Problem}

In addition to the problems facing series or shunt inductance tuning circuits which we have outlined above, both of these techniques also are subject to variations in the resonance frequency due to alignment errors between two lithographic steps, namely the definition of the SIS junction and the etching of the wiring layer. The wiring layer is used to make contact to the junction counterelectrode and to define the tuning circuit. An error in the registration of these two layers can therefore manifest itself as a variation in the length of the tuning inductor. The length of the inductor must be kept short in comparison to the propagation wavelength $\lambda_{g}$ to avoid a reduction in the bandwidth, which means that the length is usually $10 \mu \mathrm{m}$ or less for frequencies $\geq 500 \mathrm{GHz}$. Thus, a $1 \mu \mathrm{m}$ alignment error (which is a typical accuracy for a laboratory mask aligner) can cause a resonance frequency shift of $5 \%$ or more. This effect will obviously become increasingly important at higher frequencies, and may pose a serious difficulty to the production of uniformly sensitive imaging arrays. Note that this problem could be overcome if electron-beam lithography can be used to define the wiring layer.

\section{Two- Junction Tuning Circuits}

Our solution to the problems discussed above is to use a tuning circuit which incorporates two SIS junctions separated by an inductance. The circuit can either be fed from both ends as shown in Fig. 2(a), or from one end as shown in Fig. 2(b). Both of these feeding arrangements give an impedance bandwidth equal to the ideal shunt-inductance circuit in Fig. 1(a). These two-junction circuits do not use d.c. blocking capacitors, and the tuning inductance is precisely controlled by the separation of the two junctions. Since both junctions are defined in the same lithography step, the tuning inductance is nearly immune to registration errors between layers.

An SIS tuning circuit involving two junctions has been proposed previously in [15], who suggested placing the two junctions at opposite ends of a transmission line whose length is chosen so that the net junction admittance $Y=1 / Z_{j}+j \omega C_{j}$ is transformed to its complex conjugate $Y^{*}$. This would appear to be the proper condition to achieve perfect impedance match, 


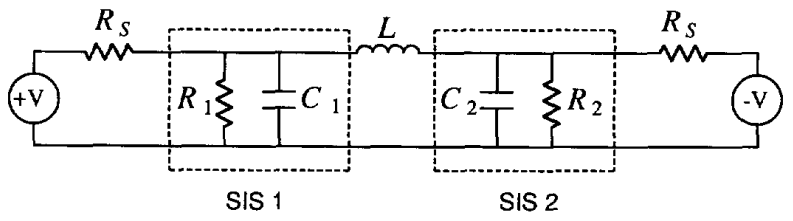

(a)

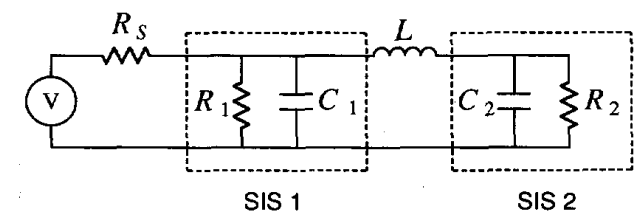

(b)

Fig. 2. Diagrams of tuning circuits which use two SIS junctions: (a) the antisymmetric "two-feed" configuration; and (b) the asymmetric "single-feed" configuration. In both circuits, the inductance is chosen so that $L / 2$ tunes out the capacitance of a single junction (assuming $C_{1}=C_{2}$ ).

but the effects of the feed circuit admittance must also be considered. When the feed circuit is included, one arrives at the correct antisymmetric feed configuration of Fig. 2(a) instead of the symmetric feed shown in [15, Fig. 2]. Recently, [16] used the asymmetric single-feed circuit (Fig. 2(b)) in a $500 \mathrm{GHz}$ quasioptical spiral-antenna mixer with excellent results, but do not discuss the issue of the power distribution between the two junctions (see subsections $\mathrm{B}$ and $\mathrm{C}$ below).

\section{A. Double-Feed Circuit}

In the first circuit (Fig. 2(a)), the signals feeding the two ends must be 180 degrees out of phase. This antisymmetric feed will produce a virtual ground at the center of the tuning inductance $L$, so each junction is effectively shunted by an inductance of $L / 2$. The circuit in Fig. 1(a) will therefore approach the full possible bandwidth, $\Delta \omega / \omega_{0}=2 /\left(\omega_{0} R_{N} C_{j}\right)$, to the extent that the inductance and the junctions behave as lumped elements. The only difficulty with this circuit is that the antisymmetric drive must be arranged somehow. Although this is straightforward in our twin-slot quasi-optical mixer, it may prove more difficult in waveguide mounts or planar antennas with only one natural feed point (e.g. a two-arm spiral).

\section{B. Single-Feed Circuit}

The operation of the single-ended tuning circuit (Fig. 2b) is most easily understood by realizing that the $C-L-C$ circuit in a $\pi$ configuration essentially behaves as a $1: 1$ impedance transformer when $\omega_{0} L / 2 \approx 1 / \omega_{0} C_{j}$. Thus, at the resonance frequency $\omega_{0}$, the impedance looking into the feed point is real and is approximately $R_{N} / 2$. However, the junctions in this single-feed circuit are not in equivalent positions as is the case for the double-feed circuit. This asymmetry manifests itself as an unequal power distribution between the two junctions for frequencies other than the resonance frequency. Fig. 3 shows that the junction closest to the feed point (SIS 1) receives a bigger fraction of the total absorbed power at frequencies above the resonance, whereas the situation is reversed below the resonance frequency. The impedance bandwidth of this

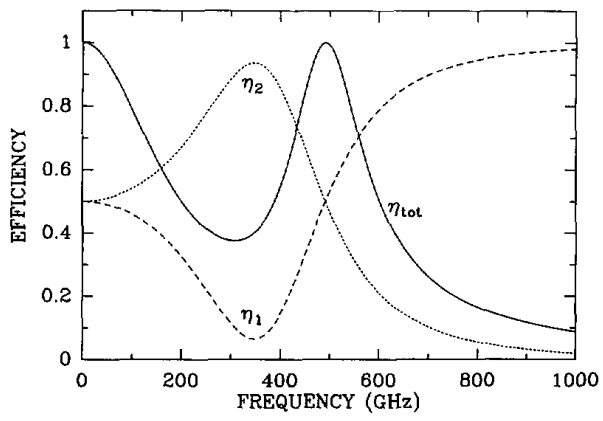

Fig. 3. This figure shows the impedance match of the asymmetric "single-feed" circuit as a function of frequency and illustrates the unequal power distribution between the two junctions. The solid line shows the impedance matching efficiency $\eta_{\mathrm{tot}}=P_{\mathrm{tot}} / P_{\text {avail }}$, which is defined asthe ratio of the total power absorbed by both junctions to the available power of the source. The dashed and dotted lines give the ratio of the power which is absorbed by each of the two junctions to the total absorbed power $\left(\eta_{1}=P_{1} / P_{\text {tot }}\right.$ and $\left.\eta_{2}=P_{2} / P_{\text {tot }}\right)$.

circuit can be defined as the frequency range for which the total power absorbed by both junctions is at least half of the power available from the generator. It is straightforward to demonstrate that this bandwidth is exactly the same as for the shunt inductance circuit shown in Fig. 1(a).

\section{Theory of the Single-Feed Circuit}

Our definition of impedance bandwidth does not take into account the mixing and noise properties of SIS junctions. Clearly, one might expect the mixer performance to degrade if one junction is better coupled to the signal source than the other, since the junction that is poorly coupled will still contribute noise to the intermediate-frequency (IF) output but it will not contribute much signal. In order to analyze this situation, we have developed a program which is based on Tucker's SIS theory [11], [12]. Tucker's theory applies to a single SIS junction, and in the three-port approximation, it characterizes a mixer in terms of a three-port admittance matrix $Y_{m m^{\prime}}$ and a noise current correlation matrix $H_{m m^{\prime}}$. Here $m=0$ refers to the IF band, $m=1$ refers to the signal sideband, and $m=-1$ refers to the image sideband. The frequencies of these bands are $\omega_{0}+m \omega$, where $\omega_{0}$ is the IF frequency and $\omega$ is the local oscillator (LO) frequency.

We can generalize Tucker's theory to the case of our twojunction circuit by treating the junctions as independent noisy three-ports and applying standard linear theory to the overall circuit. However, the matrices $Y_{m m^{\prime}}$ and $H_{m m^{\prime}}$ will not be the same for the two junctions because they depend on the LO voltage amplitude and phase, which are different for the two junctions due to the inherent asymmetry of the circuit. The matrices also depend on the I-V curves of the two SIS junctions, which can differ if the junctions are not identical.

The calculation of the LO voltage and phase for the two junctions is a nonlinear problem, but fortunately a simple solution can be obtained by choosing the LO voltage $V_{L O}^{(2)}$ on the second junction (SIS 2; the junction further from the feed) as the independent variable. Given $V_{L O}^{(2)}$, and assuming a sinusoidal LO voltage waveform (reasonable given the 
resonant tuning circuit), we can calculate the dissipative and reactive components of the LO current according to [11, (4.41)]:

$$
\begin{aligned}
\operatorname{Re} I_{L O}^{(2)}= & \sum_{n=-\infty}^{\infty} J_{n}\left(\alpha_{2}\right)\left[J_{n-1}\left(\alpha_{2}\right)\right. \\
& \left.+J_{n+1}\left(\alpha_{2}\right)\right] I_{d c}\left(V_{0}+n \hbar \omega / e\right) \\
\operatorname{Im} I_{L O}^{(2)}= & \sum_{n=-\infty}^{\infty} J_{n}\left(\alpha_{2}\right)\left[J_{n-1}\left(\alpha_{2}\right)\right. \\
& \left.-J_{n+1}\left(\alpha_{2}\right)\right] I_{K K}\left(V_{0}+n \hbar \omega / e\right),
\end{aligned}
$$

where $\alpha_{2}=e V_{L O}^{(2)} / \hbar \omega$. Since both the LO current and the LO voltage at the terminals of SIS 2 are now known, and since SIS 1 and SIS 2 are connected by a two-port passive circuit, we can immediately calculate the LO voltage on SIS 1

$$
V_{L O}^{(1)}=-\frac{I_{L O}^{(2)}+Y_{22} V_{L O}^{(2)}}{Y_{21}}
$$

where the $Y_{i j}$ are the admittance matrix elements at the LO frequency of the passive linear circuit connecting SIS 1 and SIS 2 (i.e., the tuning inductance and the junction capacitances). The LO voltage amplitude and phase calculated according to (7) allow us to calculate the three-port matrices $Y_{m m^{\prime}}$ and $H_{m m^{\prime}}$ for SIS 1 . In Tucker's theory, the LO voltage phasor is normally taken to be real, which we may assume for SIS 2 but not for SIS 1. A non-zero phase for the LO voltage can be interpreted as a time translation of the LO waveform. By considering the effect of this time translation on the signal, image, and IF waveforms, it is easy to show that an LO voltage phase shift of $\phi$ causes the three-port matrices to be modified according to

$$
Y_{m m^{\prime}}^{\prime}=Y_{m m^{\prime}} e^{i\left(m-m^{\prime}\right) \phi} \text { and } H_{m m^{\prime}}^{\prime}=H_{m m^{\prime}} e^{i\left(m-m^{\prime}\right) \phi},
$$

where $Y_{m m^{\prime}}$ and $H_{m m^{\prime}}$ are the matrices calculated with the standard expressions ([11], [12]) which assume zero LO phase. Once we have calculated the three-port admittance and noise current correlation matrices for SIS 1 and SIS 2, we simply use linear circuit theory to collapse the two-junction circuit into a single equivalent three-port circuit. We can then calculate mixer conversion loss and noise temperature from the standard expressions.

Fig. 4 shows the results of the calculation described above for the case of two identical $\mathrm{Nb} / \mathrm{Al}$-oxide/ $\mathrm{Nb}$ junctions with areas of $2 \mu \mathrm{m}^{2}$, tuned to produce a resonance near $500 \mathrm{GHz}$. The DSB mixer noise temperature as a function of frequency is plotted, and for comparison we have also shown the results of our calculation of standard single-junction mixers with shunt inductance tuning and ideal blocking capacitances of $C_{b} / C_{j}=10$ and $C_{b} / C_{j}=10^{3}$. The bias voltage and LO amplitude were optimized for minimum noise temperature at each frequency point in the calculations. Both calculations assume $C_{s}=80 \mathrm{fF} / \mu \mathrm{m}^{2}, R_{N} A=20 \Omega \mu \mathrm{m}^{2}$, a subgap to normal resistance ratio of $R_{s g} / R_{N}=10$, and a gap voltage of $V_{g}=2.9 \mathrm{mV}$ with a width of $\Delta V_{g}=0.05 \mathrm{mV}$. The RF source admittance in both calculations is taken to be real and independent of frequency, with $G_{R F}=2 / R_{N}$ for the

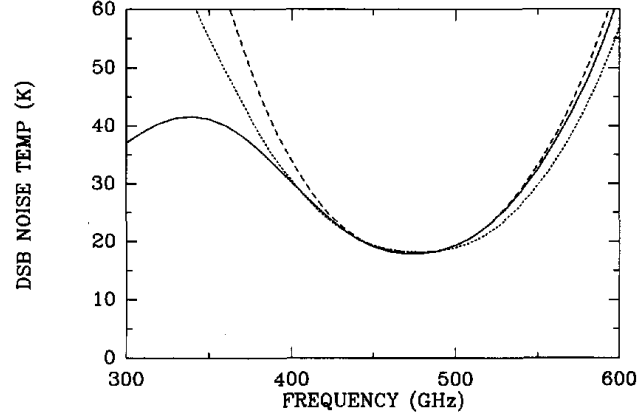

Fig. 4. The calculated noise temperature of the two-junction asymmetric "single-feed" mixer is plotted (solid line) as a function offrequency, for two identical $\mathrm{Nb} / \mathrm{Al}$-Oxide/ $\mathrm{Nb}$ junctions tuned to operate near $500 \mathrm{GHz}$ (see text for details). For comparison, we plot the calculated performance of an ideal single-junction inductively tuned mixer with $C_{b} / C_{j}=10$ (long dashes) and $C_{b} / C_{j}=10^{3}$ (short dashes). The unequal power distribution in the two-junction circuit results in a modest reduction of the bandwidth which is comparable to the effect of a finite blocking capacitance $C_{b} / C_{j}=10$.

two-junction case and $G_{R F}=1 / R_{N}$ for the single junction mixer, where $R_{N}=10 \Omega$ is the normal resistance of a single $2 \mu \mathrm{m}^{2}$ junction. The similarity of the curves in the resonance region indicates that the noise penalty for the unequal power distribution in the two-junction circuit is not severe, and is in fact no worse than the $10 \%$ bandwidth reduction caused by an ideal blocking capacitor in a single-junction circuit with $C_{b} / C_{j}=10$. Of course, in practice this capacitor may be difficult to realize as discussed in section II D.

\section{SLOT- ANTENNA MixerS WITH Two- JUNCTION TuNING CiRCUITS}

\section{A. Mixer Design}

We have fabricated and tested quasi-optical mixers with the two-junction circuits shown in Fig. 2(a) and (b), based on our twin slot antenna design [9]. Briefly, this design consists of two parallel slots of dimensions $0.5 \times 0.02 \lambda_{0}$ separated by approximately $0.3 \lambda_{0}$, where $\lambda_{0}$ is the free-space wavelength, placed on a crystal quartz substrate glued to the back of a hyperhemispherical or ellipsoidal lens.

Photographs of mixers using the single and double-feed tuning circuits are shown Fig. 5(a)-(c). The mixer in Fig. 5(a) uses a single-feed circuit (Fig. 2(b)) attached to each slot antenna and the two resulting IF signals are added in parallel, so four SIS junctions are needed in this design. For both designs, the tuning inductance is provided by a short section $\left(l / \lambda_{g}<0.1\right)$ of superconducting microstrip line. The doublefeed design (Fig. 5(c)) only requires 2 junctions since the RF signals from the two slot antennas feed this circuit from opposite sides. The antisymmetric drive (Fig. 2(a)) required for this circuit is conveniently obtained by placing radial stubs on opposite sides of the two slot antennas. These radial stubs serve to couple the slot antennas to the superconducting microstrip lines feeding the junctions.

Note that the use of a radial stub to couple to the slot antenna does not imply a significant reduction in bandwidth 


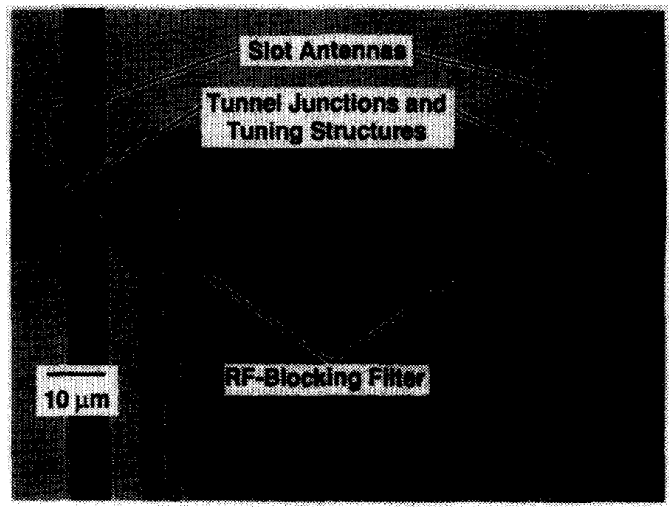

(a)

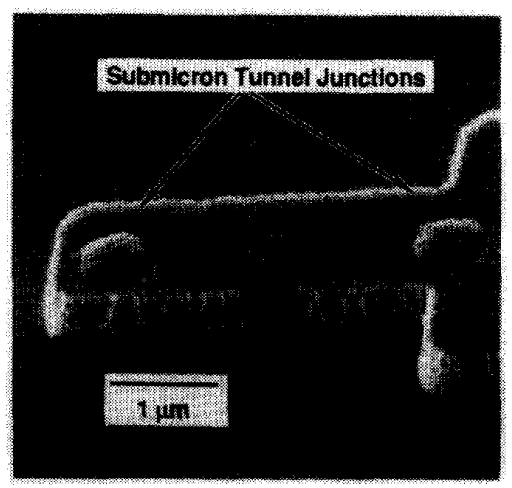

(b)

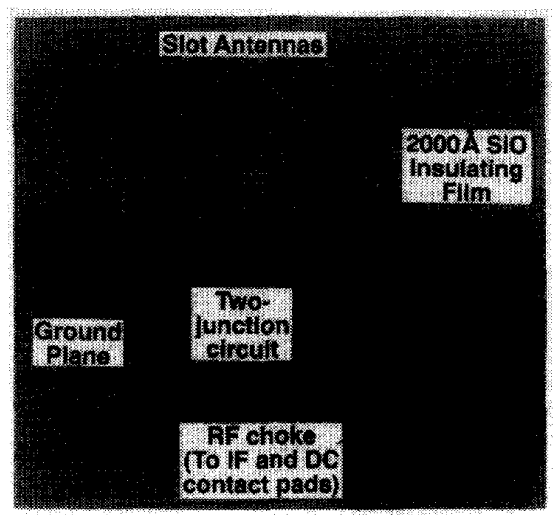

(c)

Fig. 5. Photographs of the slot antenna mixers: (a) the four-junction design which uses the asymmetric single-feed tuning circuit; a closeup of the tuning circuit used is shown in (b). Photograph (c) shows the two-junction design which uses the antisymmetric double-feed tuning circuit.

as is the case when one is used as a blocking capacitance in a SIS junction tuning circuit. Although we have verified this by direct numerical calculation, it is easy to understand if we note that we must compare the impedance of the radial stub to the impedance of the slot antenna rather than to the much lower impedance of the SIS junction capacitance.
The four-junction devices were fabricated using electronbeam lithography to define the junctions as well as the wiring layer, whereas the two-junction devices were fabricated using only optical lithography. Note that the microstrip lines feeding the junctions in Fig. $5 \mathrm{c}$ actually take the form of two quarterwavelength sections which serve to transform the $35 \Omega$ slot antenna impedance down to about $10 \Omega$. This allows $2 \mu \mathrm{m}^{2}$ area junctions to be used which are easily fabricated with optical lithography. In contrast, the four-junction device shown in Fig. 5a couples the slot antenna directly to the two-junction tuning circuit, which may be desirable at higher frequencies to avoid propagation losses in a transformer. However, this means that each junction must have a resistance of about $R_{N} \approx 70 \Omega$, which corresponds to an area of $0.3 \mu \mathrm{m}^{2}$ for our $\mathrm{Nb} / \mathrm{Al}-\mathrm{Oxide} / \mathrm{Nb}$ junctions, and so electron-beam lithography is required [17]. Furthermore, the smaller junction area implies a larger tuning inductance. The inductance can be increased by increasing the length of the microstrip inductor, but this is undesireable since the tuning bandwidth will be reduced. We have instead reduced the width of the inductor to about $1 \mu \mathrm{m}$, which means that the wiring layer must also be defined with electron beam lithography, especially because the registration with respect to the junction becomes critical. Fig. 5b shows a close-up of a two-junction circuit, which demonstrates the impressive registration accuracy $(0.1 \mu \mathrm{m})$ achievable with electron-beam lithography. For some devices, we include a single quarter-wave transformer between the junctions and the slot antenna to allow somewhat larger junctions to be used, up to about $1 \mu \mathrm{m}^{2}$. The parameters of the superconducting microstrip lines were calculated according to our previously described method [9].

There are a few interesting differences between our mixer designs and the more common single-junction designs. For instance, the beam pattern of the four-junction mixer measured in a direct-detection experiment will be that of a single slot antenna, because there is no phase relationship established between the two antennas. When the mixer is illuminated with the local oscillator field, the LO field fixes the relative phase of the two slots and thus the heterodyne beam pattern is a twin-slot pattern. Note, however, that this means that the antenna pattern will steer in the $E$-plane to look back into the direction of the incoming LO beam. This effect is also present in the two-junction mixer but at a more subtle level. If a signal is incident on the slot antennas at an angle other than normal incidence, it will not excite the two-junction circuit in a perfectly antisymmetric manner. Instead, there will be a superposition of even and odd-mode excitation. The evenmode excitation will have a voltage peak at the center of the tuning inductance rather than the virtual ground produced by the odd mode; thus, the shunt inductance tuning will not be effective for the even mode and the impedance match will be poor. The result is that the direct detection beam pattern is mainly a twin-slot pattern, with a small component of the single-slot pattern added in. Again, the imposition of an LO field establishes a definite phase relationship between the two slots, but in this case the beam-steering effect is much weaker because the relative phase of the two junctions depends only weakly on the angle of incidence of the LO because of the 


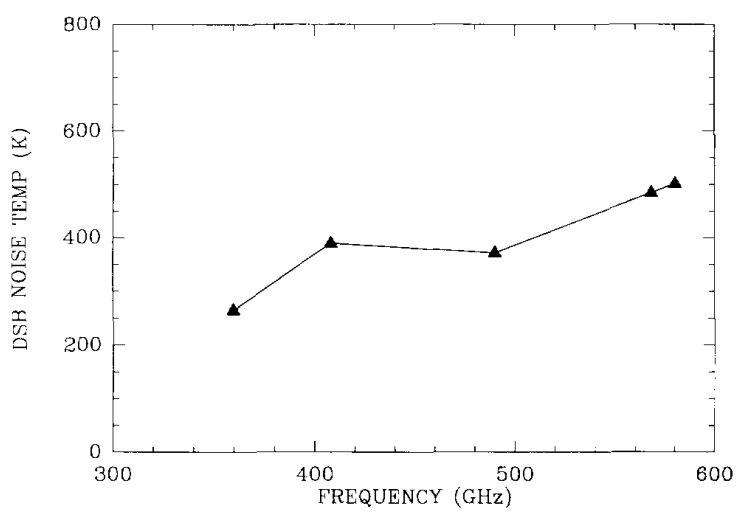

Fig. 6. The measured double-sideband receiver noise temperature of a four-junction mixer is plotted as a function of frequency.

poor even-mode coupling. Note also that an analysis along these lines will show that it is not critical to locate the twojunction circuit exactly at the center between the two slot antennas.

\section{B. Four-Junction Mixer Performance}

Fig. 6 shows the measured receiver noise temperature as a function of frequency for a four-junction mixer with $\mathrm{Nb} / \mathrm{Al}-$ Oxide/ $\mathrm{Nb}$ junctions. These results, although quite respectable, did not give the dramatic improvement we were expecting over our previous single-junction untuned mixer [9]. We are not certain what is limiting the performance of this design, but it may be that the two independent pairs of junctions were not sufficiently well matched. The fabrication of these devices was quite challenging because of the submicron dimensions, the two electron-beam steps, and the four junctions needed for each device. The yields were low, and the device quality was not as high as was typically obtained for single junctions. Nonetheless, the devices were used very successfully in a 500$600 \mathrm{GHz}$ receiver flown aboard the NASA Kuiper Airborne Observatory (KAO) in September, 1992. Fig. 7 shows a spectrum of the $\mathrm{CO}(5-4)$ transition at $576 \mathrm{GHz}$ taken towards the Orion molecular cloud OMC-1. Various other molecular and atomic lines were detected, including the ground-state transitions of $\mathrm{H}_{2}^{1 \triangleright} \mathrm{O}$ at $547 \mathrm{GHz}$ and $\mathrm{CH}$ at $532 \mathrm{GHz}$. These results confirm that the response we measure in the laboratory is indeed heterodyne detection.

\section{Two-Junction Mixer Performance}

Our difficulties with the four-junction design prompted us to consider the simpler two-junction design of Fig. 5(c). This design can be fabricated entirely with optical lithography in a simple three-step process. The fabrication process is similar to that used for the untuned mixer [9], except that only a single $2000 \AA$ thick evaporated $\mathrm{SiO}$ layer is required. The slot antennas were designed for two center frequencies, $500 \mathrm{GHz}$ and $625 \mathrm{GHz}$. Also, at each center frequency, ten different design variations were fabricated corresponding to two junction areas $\left(2\right.$ and $\left.3 \mu \mathrm{m}^{2}\right)$ and five tuning inductor lengths.

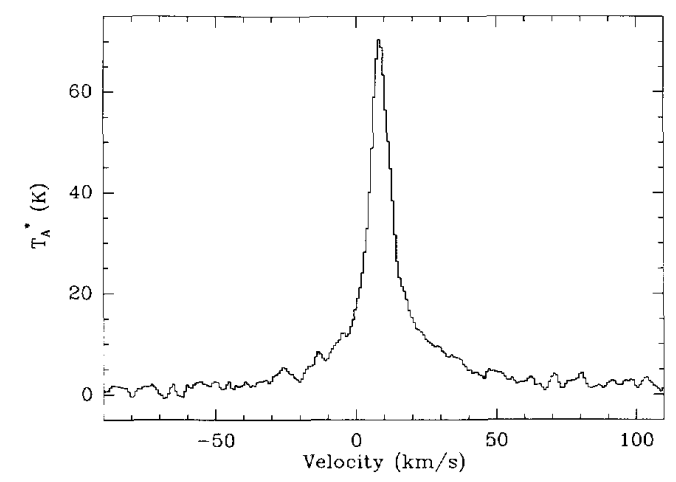

Fig. 7. A spectrum of the $576 \mathrm{GHz} J=5 \rightarrow 4$ transition of the CO molecule observed toward the Orion molecular cloud OMC- 1 from the NASA Kuiper Airborne Observatory. This spectrum was obtained in September, 1992 using an SIS receiver based on the four-junction slot antenna mixer. The integration time was two minutes.

The mixers were tested at frequencies from $360 \mathrm{GHz}$ up to $672 \mathrm{GHz}$. Solid-state local oscillators were used for all measurements. For the $500-580 \mathrm{GHz}$ band, we use a highfrequency Gunn oscillator which delivers $40 \mathrm{~mW}$ in the $125-145 \mathrm{GHz}$ band [18] followed by a Schottky varactor quadrupler [19], while the $582-672 \mathrm{GHz}$ band is covered by a $97-112 \mathrm{GHz}$ Gunn followed by a cascaded doubler-tripler combination [20]. Our results are quoted as double-sideband receiver noise temperatures and are not corrected for any optical losses (such as the LO injection beamsplitter) or for the IF noise contribution. We used a $25 \mu \mathrm{m}$ thick mylar beamsplitter $(12 \%$ reflection at $500 \mathrm{GHz})$ for all measurements, with the exception of a single point taken at 490 $\mathrm{GHz}$ with a $5 \mu \mathrm{m}$ beamsplitter. The IF bandwidth was 500 $\mathrm{MHz}$ centered at $1.5 \mathrm{GHz}$. Further details of the mixer assembly and testing are described in our previous paper [9].

Fig. 8 is a plot of the results obtained to date, with each line giving the measured receiver noise temperature vs. frequency for a given mixer chip type. Note that the bandwidth of our mixers is quite broad, often $150 \mathrm{GHz}$ or more. Our best result at $490 \mathrm{GHz}$ is a noise temperature of $113 \mathrm{~K}$ (DSB). This is equivalent to the best performance obtained with waveguide receivers in this frequency range; for instance, de Lange et al have obtained $116 \mathrm{~K}$ (DSB) at $460 \mathrm{GHz}$ [21]. At higher frequencies (and with a thicker beamsplitter), we obtain $206 \mathrm{~K}$ at $540 \mathrm{GHz}, 230 \mathrm{~K}$ at $612 \mathrm{GHz}, 269 \mathrm{~K}$ at $654 \mathrm{GHz}$, and $377 \mathrm{~K}$ at $672 \mathrm{GHz}$. The IF power curves for the $654 \mathrm{GHz}$ measurement are shown in Fig. 9. We believe that the chip to chip uniformity is very good. We tested two different chips of the same type, and found them to have nearly identical noise temperature vs. frequency curves, which is very encouraging for the development of imaging arrays. We have also used the two-junction devices in our receiver on the NASA KAO in June 1993, and obtained detections of $\mathrm{H}_{2}^{18} \mathrm{O}$ at $547 \mathrm{GHz}, \mathrm{HCl}$ at $626 \mathrm{GHz}$, and ${ }^{13} \mathrm{CO}(6-5)$ at 660 $\mathrm{GHz}$. Again, this unequivocally confirms the interpretation of our laboratory hot/cold load measurements as a heterodyne response. 


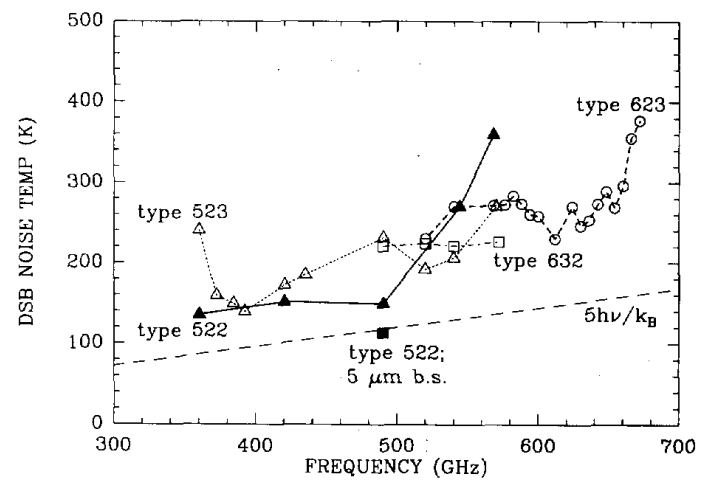

Fig. 8. The measured receiver performance for the two-junction mixer design. All of the measurements were performed with a $25 \mu \mathrm{m}$ thick mylar beamsplitter, with the exception of a single point at $490 \mathrm{GHz}$. Each line is labeled by a three-digit code giving the mixer chip type used. The first digit ( 5 or 6) gives the antenna center frequency $(500$ or $625 \mathrm{GHz})$; the second digit ( 2 or 3 ) gives the junction area $\left(2.3\right.$ or $\left.3.1 \mu \mathrm{m}^{2}\right)$; the third digit ( 1 through 5) labels the tuning inductance according to decreasing $L$.

\section{CONCLUSION}

We have demonstrated that quasi-optical SIS mixers are capable of excellent performance in the submillimeter band if a tuning circuit is used to compensate the junction capacitance. Our mixer uses a twin-slot antenna with a novel two-junction tuning circuit whose performance is equivalent to an ideal inductive shunt circuit, and which is fed in an antisymmetric fashion from two feed points. We also propose and analyze a single-feed version of this tuning circuit which is perhaps better suited to other mixer configurations such as waveguide mounts or single-terminal planar antennas.

We expect that further improvements to the mixer performance should be possible. We must study the details of the frequency dependence of the noise temperature to improve our understanding of the behavior of the tuning circuit, which would then allow further optimization. Also, the receiver currently has an estimated optical loss approaching $3 \mathrm{~dB}$ [9]. A large portion of this loss is due to the "backside" radiation of the twin-slot antenna $(26 \%)$ and the reflection loss $(15 \%)$ at the surface of the quartz hyperhemispherical lens. It may be possible to largely eliminate these losses by using an antireflection-coated silicon lens, which has a much larger dielectric constant $\left(\epsilon_{r}=11.7\right)$ and therefore a much lower backside loss $(9 \%)$. We therefore expect that the present performance can be improved by at least a factor of two in the near future.

\section{ACKNOWLEDGMENT}

The authors wish to thank G. Engargiola for assistance with the mask layout, J. Carlstrom for constructing the Gunn oscillators, D. Miller and P. Schaffer for assistance in the laboratory, and T. Büttgenbach, J. Carlstrom, J. Kooi, T. G. Phillips, R. Schoelkopf, G. Ugras, and M. Wengler for helpful discussions.

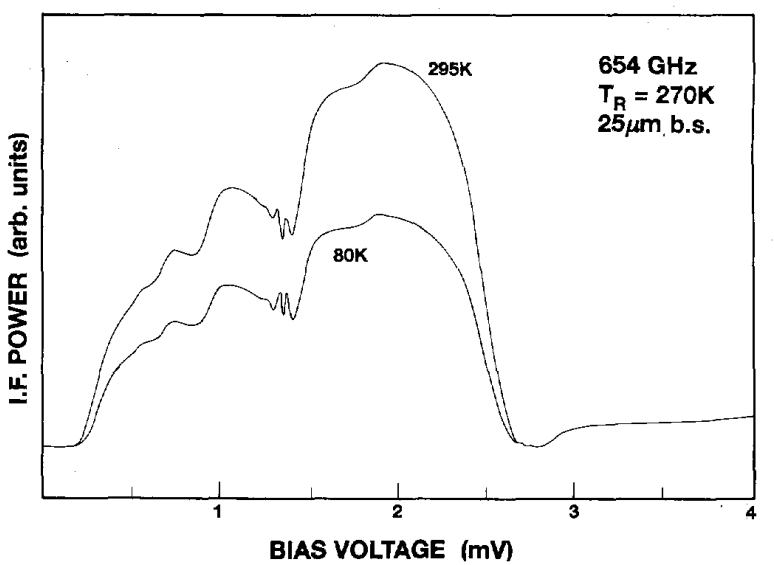

Fig. 9. Results of the laboratory measurements of the receiver sensitivity at $654 \mathrm{GHz}$. The two curves show the receiver IF output power as a function of bias voltage for hot $(295 \mathrm{~K})$ and cold $(80 \mathrm{~K})$ loads placed at the input of the receiver. A $25 \mu \mathrm{m}$ thick mylar beamsplitter was used for LO injection.

\section{REFERENCES}

[1] R. Blundell and C. E. Tong, "Submillimeter receivers for radio astronomy," Proc. IEEE, vol. 80, no. 11, pp. 1702-1720, Nov. 1992.

[2] M. J. Wengler, "Submillimeter-wave detection with superconductingtunnel diodes," Proc. IEEE vol, 80 no 11 pp. 1810-1826 Nov, 1992

[3] L. R. D'addario, "An SIS mixer for 90-120 GHz with gain and wide bandwidth," Int. J. IR and MM Waves, vol. 5, no. 11, pp. 1419-1442, 1984.

[4] A. V. Räisänen, W. R. McGrath, P. L. Richards, and F. L. Lloyd, "Broad-band RF match to a millimeter-wave SIS quasi-particle mixer," IEEE Trans. Microwave Theory Tech., vol. 33, pp. 1495-1500, Dec. 1985.

[5] M. J. Wengler, D. P. Woody, R. E. Miller, and T. G. Phillips, "A lownoise receiver for millimeter and submillimeter wavelengths," Int. J. IR and MM Waves, vol. 6, pp. 697-706, Aug. 1985

[6] T. H. Büttgenbach, R. E. Miller, M. J. Wengler, D. M. Watson, and T. G. Phillips, "A broad-band low-noise SIS receiver for submillimeter astronomy," IEEE Trans. Microwave Theory Tech., vol. 36, pp. 1720-1726, Dec. 1988

[7] Q. Hu, C. A. Mears, P. L. Richards and F. L. Lloyd, "MM wave quasioptical SIS mixers," IEEE Trans. Mag., vol. 25, pp. 1380-1383, Mar. 1989

[8] V. Y. Belitsky, M. A. Tarasov, S. A. Kovtonjuk, L. V. Filipenko, and O. V. Kaplunenko, "Low noise completely quasioptical SIS receiver for radioastronomy at $115 \mathrm{GHz}$," Int. J. IR and MM Waves, vol. 13, pp. 389-396, Apr. 1992.

[9] J. Zmuidzinas and H. G. LeDuc, "Quasi-optical slot antenna SIS mixers," IEEE Trans. Microwave Theory Tech., vol. 40, pp. 1797-1804, Sept. 1992.

[10] T. H. Büttgenbach, H. G. LeDuc, P. D. Maker and T. G. Phillips, "A fixed tuned broadband matching structure for submillimeter SIS receivers," IEEE Trans. Appl. Supercond., vol. 2, pp. 165-175, Sept. 1992.

[11] J. R. Tucker and M. J. Feldman, "Quantum detection at millimeter wavelengths," Rev. Mod. Phys., vol. 57, no. 4, pp. 1055-1113, Oct. 1985.

[12] J. R. Tucker, "Quantum limited detection in tunnel junction mixers," IEEE J. Quantum Electron, vol QE-15, pp. 1234-1258, Nov. 1979.

[13] A. R. Kerr, S.-K. Pan and M. J. Feldman, "Integrated tuning elements for SIS mixers," Int. J. IR and MM Waves, vol. 9, pp. 203-212, Feb. 1988.

[14] B. A. Syrett, "A broad-band element for microstrip bias or tuning circuits," IEEE Trans. Microwave Theory Tech., vol. MTT-28, pp. 925-927, 1980.

[15] V. Y. Belitsky and M. A. Tarasov, "SIS junction complete reactance compensation," IEEE Trans. Magn., vol. 27, pp. 2638-2641, Mar. 1991.

[16] V. Y. Belitsky, S. W. Jacobsson, L. V. Filippenko, S. A. Kovtonjuk, V. P. Koshelets et al., " $0.5 \mathrm{THz}$ SIS receiver with twin junctions tuning circuit," IEEE Microwave Guided Wave Lett., to appear. 
[17] H. G. LeDuc, B. Bumble, S. R. Cypher, A. J. Judas, and J. A. Stern, "Submicron area $\mathrm{Nb} / \mathrm{AlO}_{x} / \mathrm{Nb}$ tunnel junctions for submm mixer applications," Proc. 3rd Int. Symp. Space THz Tech., Ann Arbor, MI, Mar. 1992, pp. 408-418.

$118]$ J. E. Carlstrom Co., 262 S. Greenwood Ave., Pasadena, CA 91107

[19] Millitech Corp., P. O. Box 109, S. Deerfield, MA 01373.

[20] Radiometer Physics, Bergerwiesenstrasse 15, 5309 Meckenheim, Germany.

[21] G. de Lange, C. E. Honingh, M. M. T. M. Dierichs, H. H. A. Schaeffer, R. A. Panhuyzen, et al. "Quantum limited responsivity of a $\mathrm{Nb} / \mathrm{Al}_{2} \mathrm{O}_{3} / \mathrm{Nb}$ SIS waveguide mixer at $460 \mathrm{GHz}$," Proc. 4th Int. Symp. Space $\mathrm{THz}$ Tech., Los Angeles, CA, to appear.

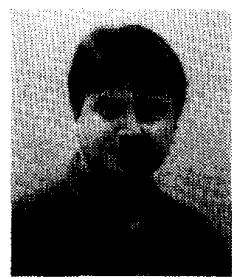

Jonas Zmuidzinas was borm September 8, 1960 in Duarte, CA. He received the B.S. degree in physics from the California Institute of Technology, Pasadena and the $\mathrm{Ph} . \mathrm{D}$. in physics from the University of California, Berkeley, in 1981 and 1987, respectively.

From 1988 to 1989, he was a Postdoctoral Fellow at the University of Illinois, Urbana, where he worked on the design and fabrication of SIS mixers. $\mathrm{He}$ is currently an Assistant Professor of Physics at the California Institute of Technology. His research interests are in the areas of high-frequency superconducting devices and its application to submillimeter astronomy.
Henry G. LeDuc was born March 8, 1955 in Butte, MT. He received the B.S. in physics from Montana State University, Bozeman, and the Ph.D. degree in physics from the University of California, Davis, in 1977 and 1983 , respectively.

Currently, he is a group leader at the Jet Propulsion Laboratory, California Institute of Technology, Pasadena. His group is developing SIS tunnel junctions for heterodyne receivers.

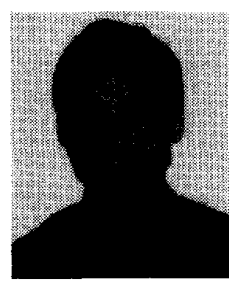

Jeffrey A. Stern received the B.S. degree in physics from Rensellaer Polytechnic Institute, Troy, NY, and the Ph.D. degree in applied physics from the California Institute of Technology, Pasadena, in 1983 and 1991 , respectively.

Currently, he is a member of the technical staff at the Jet Propulsion Laboratory, California Institute of Technology, Pasadena. His research is focused on developing $\mathrm{NbN}$ based superconductor-insulatorsuperconductor tunnel junctions for use as mixers in the submillimeter wavelength region.

Scott R. Cypher, photograph and biography, not availabe at time of publication. 